\title{
MRI and cardiac implantable electronic devices; current status and required safety conditions
}

\author{
A. W. M. van der Graaf • P. Bhagirath • M. J. W. Götte
}

Published online: 15 April 2014

(C) The Author(s) 2014. This article is published with open access at Springerlink.com

\begin{abstract}
Magnetic resonance imaging (MRI) has evolved into an essential diagnostic modality for the evaluation of all patient categories. This gain in popularity coincided with an increase in the number of implanted cardiac implantable electronic devices (CIEDs). Therefore, questions arose with regard to the MRI compatibility of these devices. Various investigators have reported the harmless performance of MRI in patients with conventional (non-MRI conditional) devices. The recently published European Society of Cardiology (ESC) guidelines on cardiac pacing and cardiac resynchronisation therapy (CRT) indicate that MRI can be safely performed in patients with an implanted pacemaker or ICD (MRI conditional or not), as long as strict safety conditions are met. This is a major modification of the former general opinion that patients with a pacemaker or ICD were not eligible to undergo MRI. This review paper attempts to elucidate the current situation for practising cardiologists by providing a clear overview of the potential life-threatening interactions and discuss safety measures to be taken prior to and during scanning. An overview of all available MRI conditional devices and their individual restrictions is given. In addition, an up-to-date safety protocol is provided that can be used to ensure patient safety before, during and after the scan. Key points

- Historically, MRI examination of patients with a CIED has been considered hazardous.

- Ongoing advances in technology and increasing usage of MRI in clinical practice have led to the introduction of MRI
\end{abstract}

The questions can be answered after the article has been published in print. You have to log in to: www.cvoi.nl.

A. W. M. van der Graaf $(\varangle) \cdot$ P. Bhagirath $\cdot$ M. J. W. Götte

Department of Cardiology, Haga Teaching Hospital, Leyweg 275,

$2545 \mathrm{CH}$ The Hague, the Netherlands

e-mail: a.vandergraaf@hagaziekenhuis.nl conditional CIEDs and to more lenient regulations on the examination of patients with non-conditional CIEDs.

- MRI investigations can be performed safely in selected patients when adhering to a standardised up-to-date safety protocol.

Keywords MRI · Cardiac implantable electronic devices · Pacing

\section{Introduction}

Historically, magnetic resonance imaging (MRI) examination of patients with a cardiac implantable electronic device (CIED) has been considered hazardous due to potential lifethreatening interaction between the MRI scanner and the pacemaker or internal cardioverter defibrillator (ICD) $[1,2]$. To increase patient safety and to anticipate the growing clinical need for MRI, an increasing number of MRI conditional CIEDs have become available [3].

However, the recently published European Society of Cardiology (ESC) guidelines on cardiac pacing and cardiac resynchronisation therapy (CRT) [4] state that MRI can be safely performed in patients with an implanted pacemaker or ICD, irrespective of the MRI conditional or non-specific MRI conditional design, as long as strict safety conditions are met.

In order to clarify this somewhat confusing situation, this review paper provides an overview of the currently available data related to CIEDs and MRI, and attempts to offer an up-todate and clinically useful summary for the practising cardiologist. In addition, a safety protocol applicable for patients with a CIED is provided that can be used to ensure a patient's safety before, during and after an MRI scan. 


\section{MRI system}

Technical background

The fundamental components of an MRI system are the main magnet coils, three gradient coils and an integral radiofrequency transmitter coil. The main magnet coils generate a strong, constant magnetic field. The strength of this magnetic field is expressed in Tesla.

Mounted inside the main magnet, three gradient coils generate gradient magnetic fields that are rapidly switched on and off. The integral radiofrequency transmitter coil produces a radiofrequency magnetic field, used to deliver energy to a population of protons. The static magnetic field and radiofrequency field combine to generate magnetic resonance signals that are spatially localised and encoded by the gradient magnetic fields [5].

Receiving coils capture the energy released by resonating hydrogen protons. Subsequent analysis results in high-grade tissue characterisation and reconstruction of a detailed image.

The amount of energy administered to an individual is expressed in SAR (specific absorption rate). SAR values vary from 2.0 to $4.0 \mathrm{~W}$ per kilogram $(\mathrm{W} / \mathrm{kg})$. For reference purposes, SAR values of a modern mobile telephone are approximately $0.9 \mathrm{~W} / \mathrm{kg}$ [6].

Over time, the strength of the static magnetic field of MRI systems has increased from less than $0.5 \mathrm{~T}$ to clinical scanners operating at $7.0 \mathrm{~T}$ and even $9.4 \mathrm{~T}$. Today, the vast majority of clinical MRI exams are performed using a $1.5 \mathrm{~T}$ system, while a rapid growth of $3.0 \mathrm{~T}$ systems is observed [7]. Since the SAR is proportional to the square of the static magnetic field strength, the amount of energy absorbed by a patient increases rapidly in a scanner operating at a higher field strength. Therefore, application of higher field strengths poses constraints on the total scan time or the imaging sequences used.

\section{Interaction between the MRI system and CIEDs}

Pavlicek et al. [8] were the first (1983) to discuss the potential interaction between pacing devices and the NMR (at that time nuclear magnetic resonance) environment using ex-vivo pacemakers of deceased patients. These interactions can be subdivided into three categories.

\section{Mechanical forces}

The constant static magnetic field strongly attracts different types of metal. Implantable devices usually contain a small amount of one or more of these metals and are considered to be ferromagnetic. Therefore, there is the possibility of movement of the implanted pacemaker.

A 6-week interval between implantation and MRI examination is advised in international guidelines in order to ensure sufficient encapsulation of the device [9].

Induction (antenna function)

The several electromagnetic components used in MRI may cause electrical or thermal induction in implanted leads. Inducted electrical currents could initiate arrhythmias or lead to oversensing or undersensing of the pacemaker or ICD with potentially fatal consequences. In addition, since tissue near the lead tip has limited conductivity, energy will be converted to heat at this location. Subsequent thermal damage around the lead tip includes oedema or formation of scar tissue. In both situations, an increase in pacing thresholds or even complete loss of capture may occur [10].

\section{Defibrillators}

The magnetic fields inside the MRI may have an unpredictable intermittent effect on the activity of the reed switch in a pacemaker or ICD. This may lead to either asynchronous pacing (reed switch closed) or unwanted inhibition of pacing in the presence of an open reed switch. In addition, the rapidly changing magnetic gradients can be registered as a life-threatening arrhythmia. The ICD can subsequently react with release of antitachycardia pacing (ATP) or shock [11]. In the presence of the main static magnetic field, the core of the transformer, necessary to charge the high-energy storage capacitor, tends to saturate. Thereby, the storage capacitor is prevented from charging. Although this reduces the risk for the deliverance of inappropriate shocks during the scan, the battery will lose life.

Software interaction

Exposure to the electromagnetic fields may also directly affect or modify the electronic circuits and functional settings of the CIED. Use of external programmers may become impossible due to damaged electrical circuits inside the CIED.

Implantable loop recorders (ILR)

Several studies have indicated that MRI scanning of ILR patients can be performed without harm to patient or device. 
However, signal artefacts that can be mistaken for a tachyarrhythmia are seen frequently $[12,13]$. Of course, image artefacts arising from the presence of an IPL can degrade the image quality.

\section{Review of literature}

Non-conditional pacemakers and ICDs

In 1984, Fetter et al. [14] investigated the potential interactions using four pacemakers in vivo. The asynchronous (VOO) pacing mode was activated during the scan in one patient. The authors concluded that patients with singlechamber implantable pacemakers may undergo scanning with MRI, provided the patient is monitored during scanning and the risks of asynchronous pacing are taken into account.

Gimbel et al. (1996) performed an MRI scan with a field strength of $0.5 \mathrm{~T}$ in five patients [15]. A 2-s pause was observed in one pacemaker-dependent patient. The other patients were asymptomatic and did not report any discomfort. Fontaine et al. (1998) demonstrated the development of an irregular ventricular rhythm in a 69-year-old patient during RF pulsing on a $1.5 \mathrm{~T}$ scanner [16]. This rhythm terminated with the cessation of RF pulsing. The patient remained asymptomatic during the procedure.

In 2000, Sommer and co-workers examined 44 nonpacemaker-dependent patients 51 times in a $0.5 \mathrm{~T}$ MRI without any impairment of pacing function [17]. Vahlhaus (2001) and co-workers postulate in a paper that 'the general policy of never exposing a patient with a pacemaker to MRI should be revised' [18].

Bartsch et al. (2003) reported four MRI-associated deaths in paced patients undergoing MRI (1.5 T) assessment [19]. Importantly, none of these patients were pacemaker dependent and in some cases ventricular fibrillation was proven to be the cause of death.

In 2005, Irnich et al. questioned 30 legal medicine departments in Germany with respect to casualties with a fatal outcome of pacemaker patients during an MRI examination (0.5-1.5 T) between 1992 and 2001 [20]. Six fatal cases occurred, in three cases ventricular fibrillation (VF) was proven to be the cause of death. In the other three cases the pacemakers were removed from the deceased patient's body and introduced in the MRI scanner. The pacemaker showed a magnetic asynchronous rate of $100 / \mathrm{min}$. It was suspected that the asynchronous pacing had induced VF in these patients.

In the following years, several investigators collected and published data on MRI examinations in patients with conventional (non-MRI conditional) pacemakers and ICDs. Naehle et al. (2009) reported the safe scanning of $18 \mathrm{ICD}$ patients at $1.5 \mathrm{~T}$ [21]. No significant change in pacing capture threshold, lead impedance or serum troponin I was observed. In two MRI examinations, oversensing of radiofrequency noise as ventricular fibrillation occurred. However, no attempt at therapy delivery was made.

More recently, Nazarian et al. (2011) published the most elaborate study [22]. A total of 555 MRI scans $(1.5 \mathrm{~T})$ were performed in 438 patients with a CIED (54\% pacemakers, $46 \%$ defibrillators). Of the $555 \mathrm{MRI}$ examinations, $222(40 \%)$ were of the brain, $122(22 \%)$ were of the spine, $89(16 \%)$ were of the heart, $72(13 \%)$ were of the abdomen or pelvis, and $50(9 \%)$ were of an extremity. Only small changes in programming, sensing and impedance were reported. The observed changes did not ever require device revision. Unfortunately, no data are available on the possible effects of repeated MRI examinations in individual patients. However, it must be noted that although a large number of different models were studied, the numbers for each individual model were small. Patients were enrolled during a long period of time (from 2003 to 2010) and CIEDs were constantly evolving. Last but not least, a control group was lacking in this study.

Despite these limitations, it is only this paper that is exclusively referred to in the recently published ESC guidelines on cardiac pacing and CRT. MRI conditional CIEDs were developed in order to decrease potential life-threatening hardware interactions.

\section{MRI conditional pacemakers and ICDs}

These systems contain specially developed components, tested and approved for usage in an MRI environment. Improved lead design reduces the risk of complications, such as lead-tip heating. In addition to the modified hardware design, MRI conditional pacemaker systems are provided with a special MRI software mode. Upon activation, the most appropriate settings are switched on automatically. These settings include bipolar stimulation instead of unipolar pacing and an increased electrical output. Specific filters inside the device prevent sensing of external non-cardiac signals. Furthermore, recording of arrhythmic episodes is temporarily disabled during the scan.

It must be noted that due to constant technological improvements the size of the available implantable pulse generators has substantially decreased and leads have become more sophisticated. Together with a reduction 
Table 1 Currently available MRI conditional pacemakers and ICDs

\begin{tabular}{|c|c|c|c|c|c|c|}
\hline & \multirow{2}{*}{\multicolumn{2}{|c|}{$\frac{\text { Medtronic }}{\text { Pacemaker }}$}} & \multirow{3}{*}{$\begin{array}{l}\text { St Jude Medical } \\
\text { Pacemaker } \\
\text { Accent MRI }\end{array}$} & \multicolumn{2}{|l|}{ Biotronik } & \multirow{3}{*}{$\begin{array}{l}\text { Boston } \\
\text { Pacemaker } \\
\text { Ingenio MRI }\end{array}$} \\
\hline & & & & \multirow{2}{*}{$\begin{array}{l}\text { Pacemaker } \\
\text { Evia ProMRI }\end{array}$} & \multirow{2}{*}{$\begin{array}{l}\text { ICD } \\
\text { Lumax ProMRI / Iforia } \\
\text { ProMRI / Ilesto ProMRI }\end{array}$} & \\
\hline & $\begin{array}{l}\text { Enrhythm MRI / } \\
\text { Revo MRI }\end{array}$ & Advisa MRI & & & & \\
\hline Restrictions & Full body & Full body & Full body & No chest scans & No chest scans & Full body \\
\hline MRI machine & \multicolumn{6}{|c|}{ Cylindrical bore magnet, clinical MRI systems with a static magnetic field of $1.5 \mathrm{~T}$} \\
\hline $\begin{array}{l}\text { SAR limitation } \\
\text { (whole body) }\end{array}$ & $\leq 2.0 \mathrm{~W} / \mathrm{kg}$ & $\leq 2.0 \mathrm{~W} / \mathrm{kg}$ & $\leq 4.0 \mathrm{~W} / \mathrm{kg}$ & $\leq 2.0 \mathrm{~W} / \mathrm{Kg}$ & $\leq 2.0 \mathrm{~W} / \mathrm{Kg}$ & $\leq 2.0 \mathrm{~W} / \mathrm{Kg}$ \\
\hline $\begin{array}{l}\text { Maximum number } \\
\text { of scans }\end{array}$ & No & No & No & $\begin{array}{l}\text { Yes: each scan } \leq 30 \text { min } \\
\text { Total scan time max: } 10 \text { hours }\end{array}$ & $\begin{array}{l}\text { Yes: each scan } \leq 30 \text { min } \\
\text { Total scan time max: } 10 \text { hours }\end{array}$ & No \\
\hline $\begin{array}{l}\text { Published clinical } \\
\text { trial evidence on } \\
\text { MRI environment }\end{array}$ & Yes & Yes & No & No & No & No \\
\hline
\end{tabular}

of ferromagnetic components in contemporary CIEDs, the chance of hardware interactions is therefore substantially diminished.

Medtronic commercialised the first MRI conditional pacemaker system (Enrhythm MRI ${ }^{\circledR}$ ) in 2008. Several trials reported the safe performance of MRI scans in patients with an implanted Enrhythm MRI system [23, 24]. However, scanning of the thorax region was prohibited. Different manufacturers initiated comparable clinical trials to demonstrate the safety of their own MRI conditional devices. Currently, several MRI conditional pacemakers are commercially available that enable full body MRI examination. Table 1 provides a complete overview of currently available MRI conditional pacemakers and ICDs.

\section{Current clinical trials}

An overview of clinical trials currently enrolling patients is given in Table 2. Apart from demonstrating the safety of a device in an MRI environment, contemporary studies are more focused on the influence on image quality and artefacts caused by the CIED (Fig. 1).

\section{Biventricular (CRT) devices}

The presence of three leads in patients treated with biventricular (CRT) therapy increases the risk of interactions between MRI and the pacing system. Only a small and inconsistent amount of literature on MRI examinations in CRT patients is available at this time. The scanning of patients with an implanted biventricular pacing device is strongly discouraged.

\section{Risk assessment}

Pacemaker dependent patients

Patients with an implanted pacemaker or ICD and an absent or insufficient intrinsic heart rhythm constitute a special group, because potential interaction between

Table 2 Current clinical trials on MRI conditional pacing devices

\begin{tabular}{llll}
\hline & Medtronic & St Jude Medical & Biotronik \\
\hline Study & Advisa II & Accent & ProMRI AFFIRM \\
Number of participating sites & 40 & 80 & 21 \\
Estimated number of patients & 270 & 800 & 245 \\
Implantable pulse generator-lead & Advisa-5076 & Accent-Tendril & Evia/Entovis-Safio S \\
Estimated date of completion & October 2014 & July 2014 & December 2013 \\
\hline
\end{tabular}


Fig. 1 Steady state free precession CINE images. Typical artefacts as can be observed in patients with an implanted impulse generator (a) and pacing leads (b). Note that the image quality in the short axis cine (b) is sufficient to allow reliable calculation of left ventricular ejection fraction

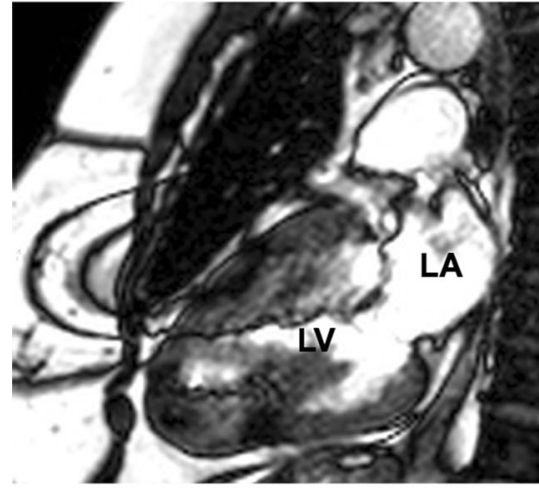

A

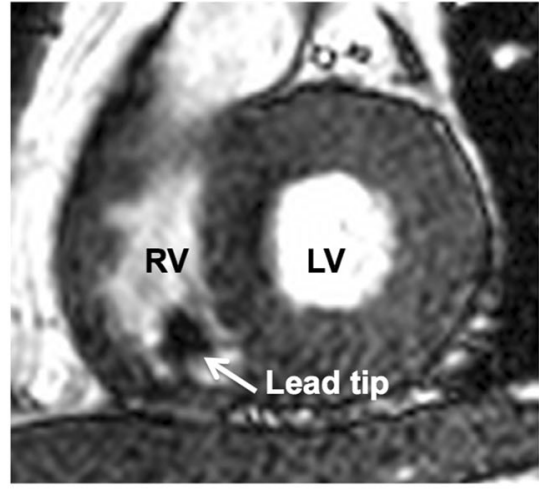

B pacemaker and MRI can have life-threatening consequences. It must be ascertained that the potential benefit of an MRI examination outweighs the potential risks and no diagnostic alternative is available. Close monitoring of the patient using pulse oximetry is warranted.

\section{MRI conditional markers and MRI mode}

Vendor-specific markers on CIEDs can facilitate the identification of MRI conditional systems on routine chest X-rays (Fig. 2). Certain CIEDs contain a selectable MRI mode, which automatically activates the most appropriate settings. After the scan, the original settings can be reprogrammed easily. Other (non-conditional) CIEDs mandate a manual selection of some of these settings and reprogramming.

Various combinations of implantable pulse generators and leads

A more complex situation occurs when implantable pulse generators and leads from different vendors, each bearing their own MRI restrictions, are combined together in individual patients. In this case the MRI compatibility of all parts of the CIED needs to be ascertained. An individualised approach is

Fig. 2 Vendor-specific markers on MRI conditional pacing systems as can be identified on routine chest X-rays. a

Medtronic, b St. Jude Medical, c Biotronik, d Boston Scientific
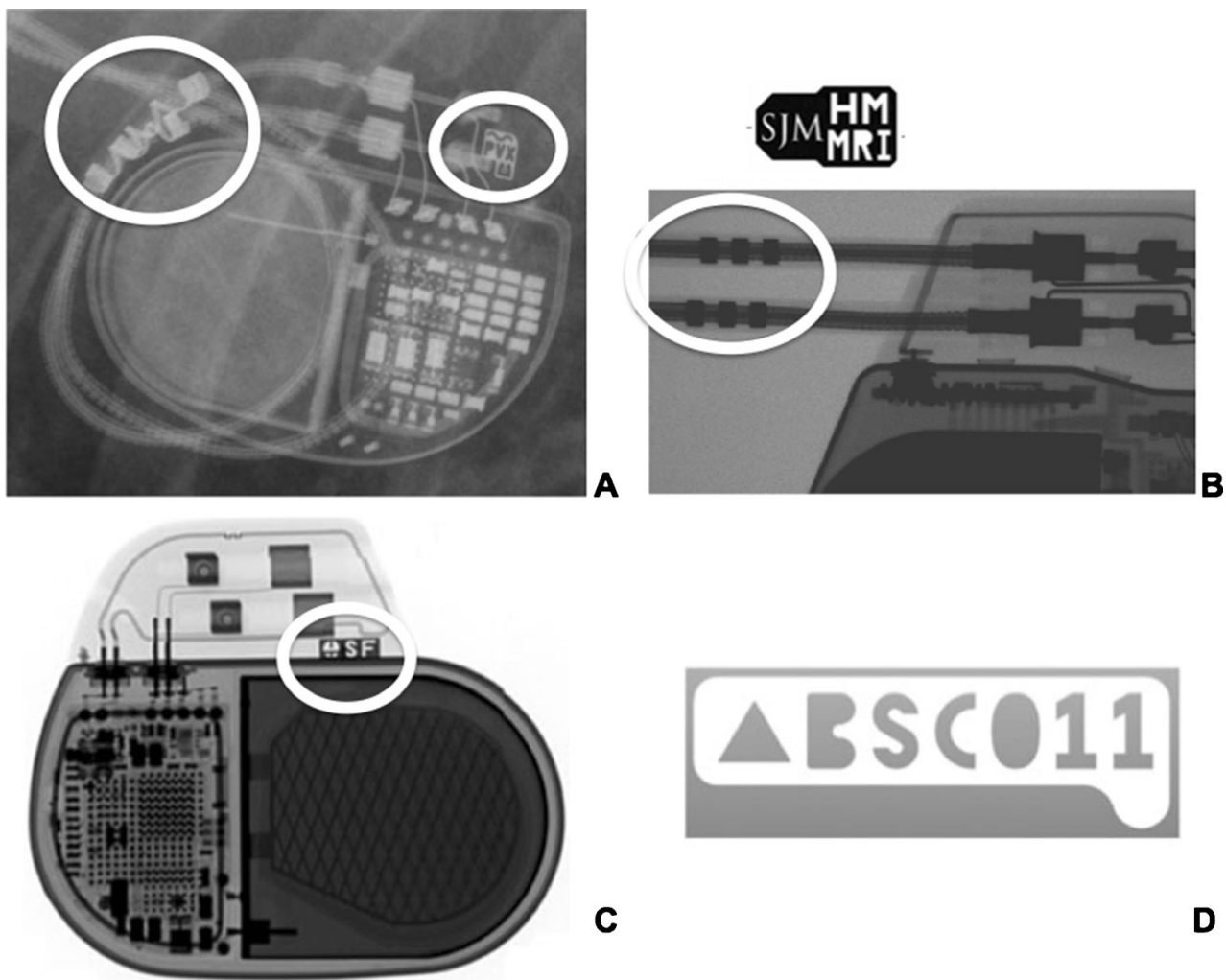

A

\section{B}

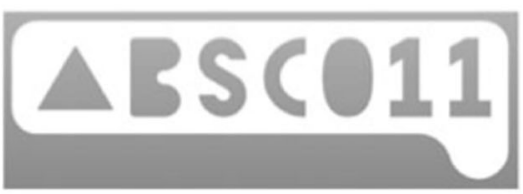

C

D 
mandatory in these patients. When abandoned pacing leads are present, for example after recent coronary bypass grafting (CABG), scanning is strongly discouraged. The absence of an implantable pulse generator increases the chance of current induction in the leads of these patients.

\section{Preparation and monitoring during MRI examination}

An MRI examination of a patient with a CIED is preferably performed exclusively in centres with extensive experience and expertise in this area. Written informed consent should be obtained from the patient after extensive notification of the procedural risks. Application of a safety protocol (Appendix 1) and appropriate monitoring are mandatory to perform an MRI scan in patients with a CIED.

Prior to the examination, proper functioning of the CIED needs to be assured. The pacing threshold is one of the most important issues and should always be determined. An already elevated stimulation threshold $(>2.0 \mathrm{~V})$ increases the risk of loss of capture during the examination. Pacemaker output should be increased and the examination should be suspended in case of a severely elevated threshold and/or deviated lead impedance $<200$ ohms or $>1,500$ ohms.

During the examination careful monitoring of the patient using a heart rate monitor and pulse oximetry is warranted. The recorded ECG signal in the scanner is subject to disturbances and therefore unreliable. Pulse oximetry is not affected by the MRI scanner and should always be used to monitor the patient. Cardiopulmonary resuscitation equipment including a cardiac defibrillator, along with staff experienced in resuscitation, should be available on site.

The nature of the cardiac MRI examination, especially in pacemaker patients, mandates routine verbal communication between operator and patient during the scan and is of vital importance. During and after the MRI examination, the patient should be asked about any discomfort or complaints. When the MRI examination is finished, the original settings of the CIED should be restored after confirming that these are still safe and provide adequate margins. In order to exclude any late side effects or symptoms, a control visit (3-6 months after the scan) to the outpatient department may be recommended [25].

\section{Discussion}

The recently published ESC guidelines on cardiac pacing and CRT somewhat trivialised the absolute necessity of MRI conditional CIEDs by stating that MRI can be safely performed in patients with an implanted pacemaker or ICD (MRI conditional or not), as long as strict safety conditions are met.

Despite abundant literature [26-31] reporting the harmless performance of MRI investigations in patients with conventional (non-MRI conditional) pacemakers and ICDs, it is still considered potentially hazardous. Only a limited number of prospective studies are available. Long-term studies are confounded by the use of several generations of CIEDs. The variety of tested devices in these studies affects the conclusions and decreases the clinical value.

Therefore, it remains difficult to state whether a specific conventional CIED can be introduced into the MRI room without possible consequences. For MRI conditional CIEDs, the possibilities, limitations and required safety measures to be taken are more uniform and described in detail.

In daily practice, an increasing number of clinicians are confronted with questions on MRI compatibility of CIEDs. The value of a safety protocol, approved by the cardiology and radiology department, cannot be stressed enough.

It is expected that the role of MRI in clinical decisionmaking will gain even more clinical importance. There is an emerging role for MRI in identifying arrhythmogenic substrates and this modality has an expanding role in guiding electrophysiological therapies.

Now that the patient's safety seems to be conditionally guaranteed, a new challenge is the reduction of the impact of CIEDs on the image quality, especially with regard to the identification and modification of an arrhythmogenic substrate.

Finally, it is important to realise that almost all published data are only valid for scanning on 1.5 Tesla machines. The application of 3.0 Tesla machines is rapidly emerging, especially for orthopaedic and neurological patients, due to the higher signal-to-noise ratio (SNR). With the advance of $3.0 \mathrm{~T}$ machines, new technical challenges arise.

\section{Conclusion}

MRI conditional CIEDs are designed to cope with the challenges introduced by the electromagnetic MRI environment. Various papers have demonstrated the safe and harmless performance of MRI examination in patients with CIEDs, MRI conditional or not. With appropriate monitoring and application of a safety protocol, MRI can be safely performed in patients with CIEDs. For patients equipped with a conventional CIED or those who are pacing dependent it must be ascertained that the potential benefits of an MRI examination outweigh the potential risks.

Disclosures Our institution has received research grants from St Jude Medical and Medtronic NL. None of the authors report a potential conflict of interest.

Open Access This article is distributed under the terms of the Creative Commons Attribution License which permits any use, distribution, and reproduction in any medium, provided the original author(s) and the source are credited. 


\section{Appendix 1}

\section{CIED - MRI protocol}

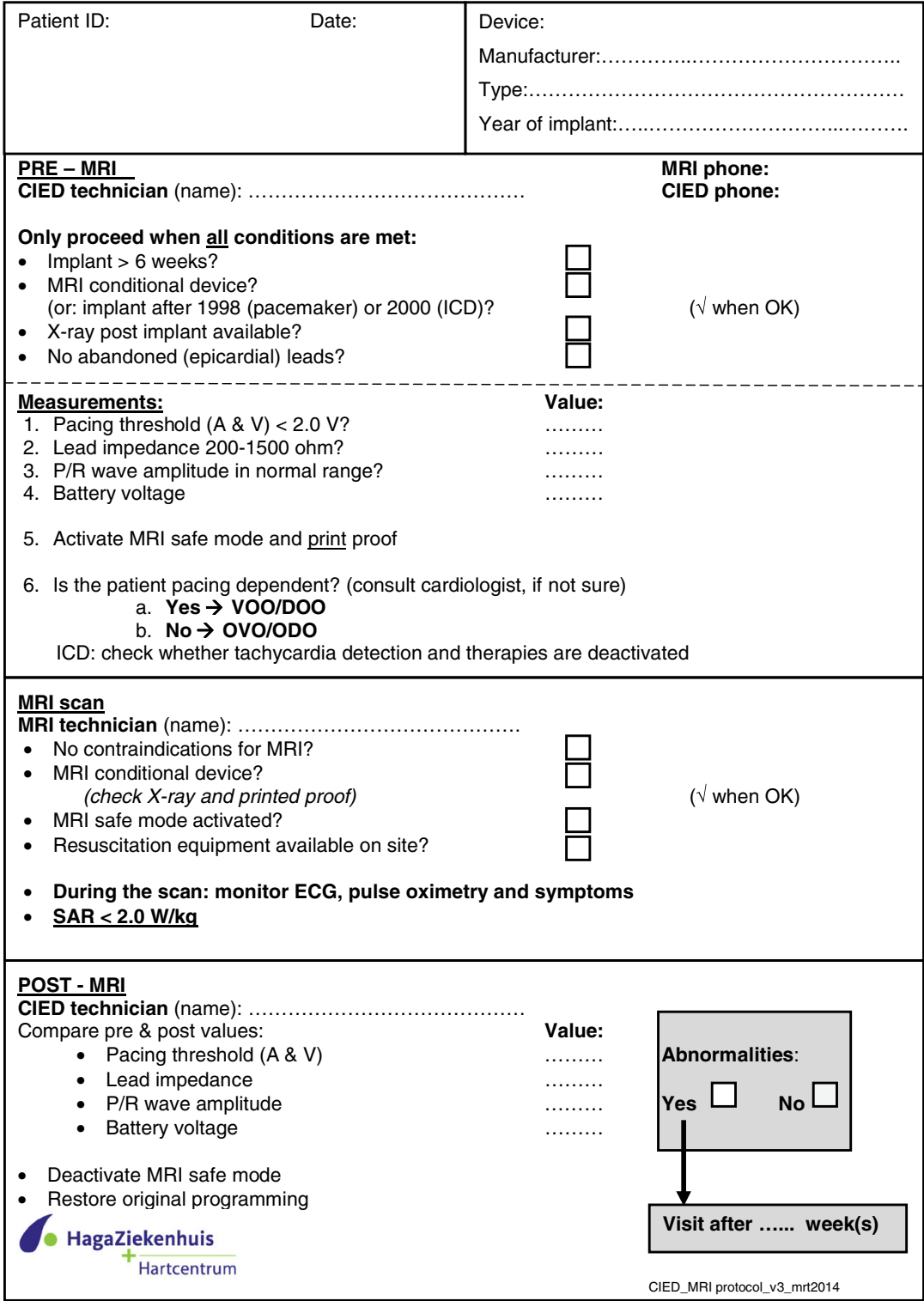

\section{References}

1. Kalin R, Stanton MS. Current clinical issues for MRI scanning of pacemaker and defibrillator patients. Pacing Clin Electrophysiol. 2005;28:326-8.

2. Langman DA, Goldberg IB, Finn JP, et al. Pacemaker lead tip heating in abandoned and pacemaker-attached leads at 1.5 Tesla MRI. J Magn Reson Imaging. 2011;33:426-31.

3. Ahmed FZ, Morris GM, Allen S, et al. Not all pacemakers are created equal: MRI conditional pacemaker and lead technology. J Cardiovasc Electrophysiol. 2013;24(9):1059-65.

4. Brignole M, Auricchio A, Baron-Esquivias G, et al. ESC guidelines on cardiac pacing and cardiac resynchronization therapy: the task force on cardiac pacing and resynchronization therapy of the European Society of Cardiology (ESC). Developed in collaboration with the European Heart Rhythm Association (EHRA). Europace. 2013;15(8):1070-118.

5. Ridgway JP. Cardiovascular magnetic resonance physics for clinicians: part 1. J Cardiovasc Magn Reson. 2010;12:71.

6. Götte MJW, Rüssel IK, de Roest GJ, et al. Magnetic resonance imaging, pacemakers and implantable cardioverter-defibrillators: current situation and clinical perspective. Neth Heart J. 2010;18:31-7.

7. van der Wall EE. Crown years for non-invasive cardiovascular imaging (Part III): 30 years cardiovascular magnetic resonance. Neth Heart J. 2013;21(6):263-5.

8. Pavlicek W, Geisinger M, Castle L, et al. The effects of nuclear magnetic resonance on patients with cardiac pacemakers. Radiology. 1983;147(1):149-53.

9. Luechinger R, Duru F, Scheidegger MB, et al. Force and torque effects of a 1.5 Tesla MRI scanner on cardiac pacemakers and ICD’s. Pacing Clin Electrophysiol. 2001;24:199-205. 
10. Luechinger R, Zeijlemaker VA, Pederson EM, et al. In vivo heating of pacemaker leads during magnetic resonance imaging. Eur Heart J. 2004;26:376-83.

11. Ainslie M, Miller C, Brown B, et al. Cardiac MRI of patients with implanted electrical cardiac devices. Heart. 2013;0:1-7. doi:10.1136/ heartjnl-2013-304324.

12. Gimbel JR, Zarghami J, Machado C, et al. Safe scanning, but frequent artifacts mimicking bradycardia and tachycardia during magnetic resonance imaging (MRI) in patients with an implantable loop recorder (ILR). Ann Noninvasive Electrocardiol. 2005;10(4):404-8.

13. Wong JA, Yee R, Gula LJ, et al. Feasibility of magnetic resonance imaging in patients with an implantable loop recorder. Pacing Clin Electrophysiol. 2008;31(3):333-7.

14. Fetter J, Aram G, Holmes DR, et al. The effects of nuclear magnetic resonance imagers on external and implantable pulse generators. Pacing Clin Electrophysiol. 1984;7:720-7.

15. Gimbel JR, Johnson D, Levine PA, et al. Safe performance of magnetic resonance imaging on five patients with permanent cardiac pacemakers. Pacing Clin Electrophysiol. 1996;19(6):913-9.

16. Fontaine JM, Mohamed FB, Gottlieb C, et al. Rapid ventricular pacing in a pacemaker patient undergoing magnetic resonance imaging. Pacing Clin Electrophysiol. 1998;21:1336-9.

17. Sommer T, Vahlhaus $C$, Lauck G, et al. MRI and cardiac pacemakers: in-vitro evaluation and in vivo studies in 51 patients at $0.5 \mathrm{~T}$. Radiology. 2000;215:869-79.

18. Vahlhaus C, Sommer T, Lewalter T, et al. Interference with cardiac pacemakers by magnetic resonance imaging: are there irreversible changes at 0.5 T? Pacing Clin Electrophysiol. 2001;24:489-95.

19. Bartsch C, Irnich W, Risse M, Weiler G. Unexpected sudden death of pacemaker patients during or shortly after magnetic resonance imaging [MRI]. In: XIX Congress of International Academy of Legal Medicine. Milan, Italy, 3-6 September 2003. 174, Abstract no. 114

20. Irnich W, Irnich B, Bartsch C, et al. Do we need pacemakers resistant to magnetic resonance imaging? Europace. 2005;7(4):353-65.
21. Naehle CP, Strach K, Thomas D, et al. Magnetic resonance imaging at 1.5-T in patients with implantable cardioverter-defibrillators. J Am Coll Cardiol. 2009;54(6):549-55.

22. Nazarian S, Hansford R, Roguin A, et al. A prospective evaluation of a protocol for magnetic resonance imaging of patients with implanted cardiac devices. Ann Intern Med. 2011;155:415-24.

23. Sutton R, Kanal E, Wilkoff BL, et al. Safety of magnetic resonance imaging of patients with a new Medtronic EnRhythm MRI SureScan pacing system: clinical study design. Trials. 2008;9:68.

24. Wilkoff BL, Bello D, Taborsky M, et al. Magnetic resonance imaging in patients with a pacemaker system designed for the magnetic resonance environment. Heart Rhythm. 2011;8(1):65-73.

25. Nazarian S, Beinart R, Halperin HR. Magnetic resonance imaging and implantable devices. Circ Arrhythm Electrophysiol. 2013;6(2): 419-28.

26. Cronin EM, Mahon N, Wilkoff BL. MRI in patients with cardiac implantable electronic devices. Expert Rev Med Devices. 2012;9(2): $139-46$.

27. Cohen JD, Costa HS, Russo RJ. Determining the risks of magnetic resonance imaging at 1.5 tesla for patients with pacemakers and implantable cardioverter defibrillators. Am J Cardiol. 2012;110:1631-6.

28. Boilson BA, Wokhlu A, Acker NG, et al. Safety of magnetic resonance imaging in patients with permanent pacemakers: a collaborative clinical approach. J Interv Card Electrophysiol. 2012;33:59-67.

29. Coman JA, Martin ET, Sandler DA, et al. Implantable cardiac defibrillator interactions with magnetic resonance imaging at 1.5 tesla. J Am Coll Cardiol. 2004;43:138A.

30. Gimbel JR, Kanal E, Schwartz KM, et al. Outcome of magnetic resonance imaging (MRI) in selected patients with implantable cardioverter defibrillators (ICDs). Pacing Clin Electrophysiol. 2005;28:270-3.

31. Coman JA, Martin ET, Ramza BM, et al. Pacemaker safety during magnetic resonance imaging at 1.5 Tesla. J Am Coll Cardiol. 2001;37(Suppl A):436.

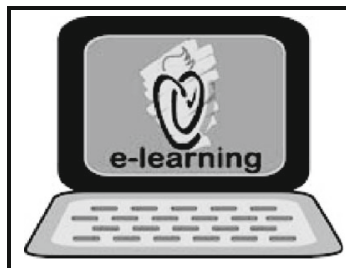

\section{CVOI E-learning formula!}

This is the CVOI e-learning article. The author has prepared 10 questions which are available through the website of the Cardiovascular Educational Institute (CVOI). Please follow the instructions below.

After finishing the questions you will be asked to fill in your name, hospital and e-mail address; then press the button 'verzenden'.

When 6 out of the 10 questions are answered correctly, you acquire 1 accreditation point granted by the Quality Committee of the Netherlands Society of Cardiology (NVVC). The acquired point will be credited to your personal file in the GAIA system. You will also receive an e-mail with all the correct answers.

Over a period of one year 10 e-learning articles will appear in 10 subsequent NHJ editions. In each edition the e-learning article will be recognisable by a special icon. On an annual basis you can collect 10 accreditation points. The accreditation points are credited in the GAIA system by the CVOI.

If you need additional information, please contact the CVOI by e-mail: cvoi@cvoi.org or by phone: 030-2345001.

E.E. van der Wall

Chief editor NHJ
K.B. Schick

Coordinator CVOI 